\title{
Gretchenfrage: Hilft bessere Bezahlung gegen den Pflegemangel?
}

\section{Hauptstadtkongress Medizin und Gesundheit}

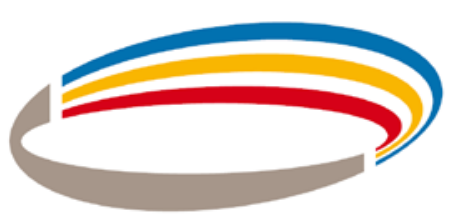

HAUPTSTADT
KONGRESS 2017

» Während Fachkräfte aus Kundenservice und Logistik im Durchschnitt mehr als 39.000 Euro brutto verdienen, bekommt ein Altenpfleger nur 24.657 Euro. Aber nicht einmal überall: Der Verdienst in der Pflege schwankt je nach Bundesland um bis zu 35\%. Die Folgen kann man in Sachsen sehen: Dort werden zwar fast doppelt so viele Pflegekräfte ausgebildet wie notwendig, aber das reicht nicht. Viele Absolventen wandern aus Sachsen ab, wechseln den Beruf oder gehen auf Teilzeit. Mancherorts gibt es bereits Aufnahmestopps und Bettenstilllegungen in Pflegeeinrichtungen.

Karl Brenke vom Deutschen Institut für Wirtschaftsforschung sähe Abhilfe in besserer Bezahlung, beklagt aber: „Wenn Fluglotsen knapp sind, steigen die Löhne relativ stark und über Streiks können sie höhere Gehälter fordern. Altenpfleger hingegen sind weniger gewerkschaftlich organisiert. Da wird Fachkräftemangel beklagt, aber es fehlt die Macht, bessere Löhne durchzusetzen." Das sieht der Patientenbeauftragte der Bundesregierung, KarlJosef Laumann, ähnlich: Über Jahrzehnte hätten die Krankenhäuser auf Kosten der Pflege gespart, während die Ärzte hohe Tariferhöhungen erkämpft hätten. Sylvia Bühler von der Gewerkschaft ver.di fordert nun: „Dass viele Arbeitgeber die Empathie der Beschäf- tigten und ihr großes Engagement so schamlos ausnutzen können, muss aufhören!" Die jedoch halten dagegen: Die von ver.di geforderte Anhebung des Pflegemindestlohns auf 12,50 Euro nannte der Präsident des bpa Arbeitgeberverbands, Rainer Brüderle, „Cockpit-Niveau“. Auf dem Hauptstadtkongress diskutieren Karl Brenke vom DIW, Dietmar Erdmeier von ver.di, Kai A. Kasri vom Altenpflegeunternehmen Vivaldo, Rupert Niewiadomski von der Katholischen Sozialstation Freiburg und Rainer Brüderle, Präsident des bpa Arbeitgeberverbands das Thema.

Der Hauptstadtkongress Medizin und Gesundheit ist mit mehr als 8.000 Entscheidern aus Gesundheitswirtschaft und Politik die jährliche Leitveranstaltung der Branche. Er findet vom 20. bis 22. Juni 2017 im CityCube Berlin statt.

www.hauptstadtkongress.de

\section{Qualität neu definieren: Patienten, Personal und Gemeinwohl}

\section{Christliche Krankenhausverbände positionieren sich zur Bundestagswahl}

" Die Initiative „Christliche Krankenhäuser in Deutschland“ (CKiD) fordert eine entschlossene Ausrichtung der Krankenhauspolitik der kommenden Legislaturperiode auf der Basis eines erweiterten Qualitätsverständnisses. Priorität sollen dabei die Patienten, das Personal und das Gemeinwohl haben. Drängender Handlungsbedarf bestehe hinsichtlich der Neuregelung von Personaluntergrenzen und bei der flächendeckenden Notfallversorgung. Auch die Versorgung vulnerabler Patienten müsse verbessert werden, so der Deutsche Evangelische Krankenhausverband (DEKV) und der Katholische Krankenhausverband Deutschlands (kkvd). Die Regelungen des Krankenhausstrukturgesetzes (KHSG) reichten nicht aus, eine sowohl effektive als auch durch Menschlichkeit und $\mathrm{Zu}$ wendung geprägte Patientenversorgung sicherzustellen.

„Wir setzen uns für ein neues Verständnis von Qualität in der Krankenhausbehandlung ein, das sich deutlich an patienten- und gemeinwohlbezogenen sowie professionsethischen Kriterien orientiert. Ein umfassender Qualitätsbegriff muss auch den einzelnen Patienten im Blick haben und sollte sich außerdem stärker an Indikatoren zur Messung der Lebensqualität wie beispielsweise die International Classification of Functioning (ICF) ausrichten", sagt der DEKV-Vorsitzende Christoph Radbruch. Ein Umdenken sei insbesondere im Umgang mit demenzkranken oder geistig behinderten Patienten notwendig. Um die patientenorientierte Versorgung zu stärken, sei es zudem notwendig, ausreichend Pflegepersonal auszubilden. „Unverzichtbar hierfür ist eine eigenständige, bundesweit geregelte zweijährige Assistenzausbildung, die den Erfordernissen am Arbeitsmarkt entspricht und attraktive Weiterbildungsmöglichkeiten für die Absolventen eröffnet."

kkvd-Vize Ingo Morell betont: „Die Politik ist in der kommenden Legisla- turperiode gefordert, auf die reale Situation in der Versorgung zu schauen - mit allen regionalen Besonderheiten und personellen Engpässen." Das aktuelle Finanzierungssystem stoße auf Grund der höheren Anzahl von multimorbiden Patienten und den dadurch bedingten höheren Anforderungen an die Pflegenden, an seine Grenzen. Daher fordere die CKiD eine vorausschauende und realistische Planung sowie eine Refinanzierung, die den Krankenhäusern ermögliche, eine zukunftsfeste Versorgung zu gestalten. Zum Thema Notfallversorgung sagt Morell: „Es muss endlich Schluss sein mit der sektoralen Abgrenzungsdiskussion."

www.christliche-krankenhaeuser.de 